\title{
Comparação das respostas fisiológicas durante a prática de exergame e atividades convencionais: uma revisão sistemática com metanálise
}

\author{
Comparison of physiological responses during practices of exergames \\ and conventional activities: a systematic review and meta-analysis
}

Breno Berny Vasconcelos; Andressa Formalioni'; Leony Morgana Galliano; César Augusto Otero Vaghetti'; Fabrício Boscolo Del Vecchio ${ }^{1}$

\begin{abstract}
Resumo
O objetivo do presente trabalho foi realizar revisão sistemática com metanálise dos estudos sobre efeitos fisiológicos da prática de exergames, a fim de compará-las quando realizadas de maneira convencional. As buscas foram realizadas nas bases eletrônicas: PubMed, Science Direct, Google Scholar e a revista Games For Health Journal, utilizando os seguintes descritores ou termos: "bealth video game" OR "active video game" AND "energy expenditure"; exergam* AND "physical activity"; exergam* AND comparison; exergam* compared to real; exergam* AND "real game"; exergam* AND "real sports". Para serem incluídos, além de serem originais, os artigos também deveriam comparar a realização das atividades convencionais igualmente efetivadas com exergame (EXG). Foram encontrados 2.928 estudos que abordaram a temática. Após avaliação por títulos, 13 artigos foram excluídos por serem duplicatas. No total foram lidos 20 resumos, no entanto, selecionamos apenas sete para entrar na revisão. Além disso, três estudos localizados nas listas de referências foram incluídos. Os estudos que passaram pelos filtros de análise acabaram submetidos à escala PEDro, para mensuração de qualidade metodológica. A metanálise apontou não haver diferença significativa, entre atividades com EXG e atividades convencionais, para frequência cardíaca $(\mathrm{p}=0,248)$, percepção subjetiva de esforço $(\mathrm{p}=0,295)$, gasto energético ( $\mathrm{p}$ $=0,664)$ e consumo de oxigênio $(p=0,455)$. Desta maneira, conclui-se que não há diferença para as variáveis apresentadas em ambas as atividades propostas.
\end{abstract}

\section{Palavras-chave}

Metanálise; Atividade motora; Jogos de vídeo; Metabolismo energético; Consumo de oxigênio.

\begin{abstract}
The aim of this study was to perform a systematic review and metanalysis on the physiological effects of exergames practice compared to the same activities performed in a conventional manner. Therefore, a search was made in the databases PubMed, Science Direct, Google Scholar and Games For Health magazine using the following descriptors or terms "bealth video game" oR "active video game" AND "energy expenditure"; exergam * AND "physical activity"; exergam * AND comparison; exergam * Compared to real; exergam * AND "real game"; exergam * AND "real sports". To be included in the review, studies should be original articles and rely on activities performed with active videogames, namely exergames (EXG), which should be compared with the same conventional activities. An amount of 2928 studies were found. After title evaluation, 13 studies were excluded for duplication, lasting 20 titles. After abstract's evaluation, 7 titles were selected for entering this study. Besides, 3 other studies were found in reference lists and were included.Studies passed by search filters were submitted to PEDro scale for methodological quality evaluation. Metanalysis showed no significant differences for heart rate $(p=0.248)$, rating of perceived exertion ( $p=0.295)$, energy expenditure $(p=0.664)$ and oxygen uptake $(p=0.455)$ between EXG and conventional activities. In conclusion, there are no differences between activities with EXG and the same activities realized in a conventional manner on heart rate, rate of perceived exertion, energy expenditure and oxygen consumption.
\end{abstract}

\section{Keywords}

Meta-analysis; Motor activity; Video games; Energy expenditure; Oxygen consumption.

\section{Introdução}

Vídeo games ativos, conhecidos como exergames (EXG), são aqueles nos quais os jogadores intera- gem com o jogo a partir de movimentos corporais amplos ${ }^{1}$. Por estimularem movimentações corporais semelhantes às de atividades físicas convencionais, os EXG vêm sendo utilizados como alternativa para aumentar a prática de atividades físicas em crianças, 
adolescentes, adultos e idosos ${ }^{2-6}$. No entanto, o conhecimento escasso sobre a comparação da intensidade das práticas em EXG em relação às atividades convencionais demandam estudos sobre o tema e sumarização do conteúdo já existente.

Diversas investigações com EXG conduzidas nos últimos anos envolveram aplicação desta tecnologia para aumentar a adesão de escolares nas aulas de Educação Física ${ }^{3}$, melhorar o equilíbrio em idosos ${ }^{4}$ e investigar os seus efeitos fisiológicos $^{5,6}$. Vários estudos compararam os efeitos dos EXG sobre variáveis fisiológicas (frequência cardíaca, concentração de lactato sanguíneo e consumo de oxigênio) com jogar um videogame convencional (não ativo), correr na esteira e até mesmo atividades semelhantes às realizadas nos jogos, como a dança ${ }^{7}$. Todavia, não há sumarização do perfil coletivo dos achados destes estudos isolados.

Previamente, indicou-se que a prática de alguns EXG proporciona intensidade suficiente para atender às recomendações do American College of Sports Medicine ${ }^{8}$ de atividades físicas de nível moderado para adultos ${ }^{9,10}$. Porém, devido à extensa variedade metodológica dos estudos existentes, pouco se sabe sobre quão próximos são os efeitos fisiológicos do jogo em EXG em relação às mesmas atividades realizadas de forma convencional. Hipotetiza-se que, apesar das atividades apresentarem demandas semelhantes, possam existir efeitos distintos, principalmente devido aos níveis de imersão e engajamento que tendem a ser mais altos em EXG, ao compará-los com atividades físicas convencionais ${ }^{11}$. Portanto, o objetivo deste estudo foi realizar uma revisão sistemática e metanálise sobre os efeitos fisiológicos agudos entre essas práticas de atividades descritas.

\section{Métodos}

Este estudo se caracteriza como uma revisão sistemática da literatura com metanálise, a qual foi estruturada a partir das diretrizes do PRISMA ${ }^{12}$. As buscas foram realizadas por um dos pesquisadores, e ocorreram nas seguintes bases de dados: PubMed, Science Direct e Google Scholar. Adicionalmente, foram revisados artigos publicados na revista Games For Health, dada sua relevância para a temática, além de buscas nas listas de referências dos estudos selecionados nas bases de dados já citadas. Para o processo de busca dos dados, empregaram-se as seguintes combinações dos descritores ou termos, que foram realizadas de modo independente: i) "bealth video game" OR "active video game" AND "energy expenditure"; ii) exergam* AND "physical activity"; iii) exergam* AND comparison; exergam* compared to real; iv) exergam* AND "real game"; v) exergam* AND "real sports".

Os artigos experimentais originais incluídos contam com atividades realizadas com mediação tecnológica (EXG), as quais precisariam ser comparadas com as mesmas atividades convencionais. Excluíram-se artigos que não apresentassem resultados relacionados com comparações diretas de variáveis metabólicas ou fisiológicas em situação de convencional e com EXG. As buscas realizaram-se em maio de 2016 sem restrição de data de publicação e população alvo. As etapas de busca foram: análise de títulos, análise de resumos, análise de artigos na íntegra e análise das listas de referências. Após selecionados, foram submetidos à escala PEDro (Physiotherapy Evidence Database) ${ }^{13}$, utilizada apenas para caracterizar a qualidade metodológica dos estudos incluídos à revisão, e não como um critério de exclusão.

Os dados de cada um dos estudos foram tabulados em planilha do software Microsoft Excel ${ }^{\circledR}$ (pacote Microsoft Office ${ }^{\circledR}$ versão 2013, desenvolvido pela Microsoft $\left.{ }^{\circledR}\right)$ com informações sobre o autor, ano de publicação, características da amostra, número amostral, média e desvio padrão de cada uma das variáveis fisiológicas analisadas. A análise estatística dos dados foi realizada no programa 
estatístico Stata versão 12.0, através do teste de efeitos aleatórios para dados contínuos. Foram utilizados o "n" dos grupos, as médias e desvios-padrão de gasto energético considerando EXG e exercício convencional. A heterogeneidade estatística entre os estudos foi avaliada através dos testes $\mathrm{Q}$ de Cochran e $\mathrm{I}^{2}$.

\section{Resultados}

Foram encontrados 2.928 estudos potencialmente relevantes. Na avaliação dos títulos, foram identificadas 33 publicações referentes à temática da revisão e 13 artigos foram excluídos por duplicação em bases de dados distintas. Dos 20 estudos restantes, com base na leitura dos resumos, sete atenderam aos critérios de inclusão, foram lidos na íntegra. Avaliando integralmente os sete estudos restantes, todos foram selecionados para entrar na revisão. Analisando as listas de referências, três estudos foram encontrados e incluídos à revisão, compondo total de 10 artigos incluídos. O processo de seleção dos artigos está detalhado no diagrama de fluxo (Figura 1).
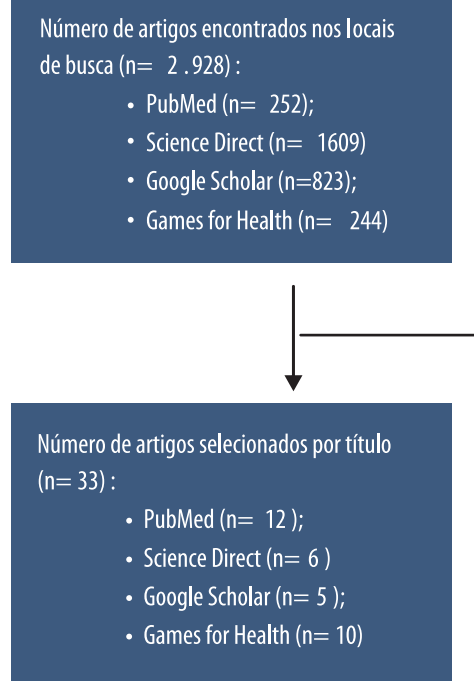

Número de artigos excluídos por título, por não abordarem o tema especifico $(n=2895)$ :

PubMed $(n=240)$;

- Science Direct $(n=1603)$

Google SC holar $(n=818)$ Games for Health $(n=234)$
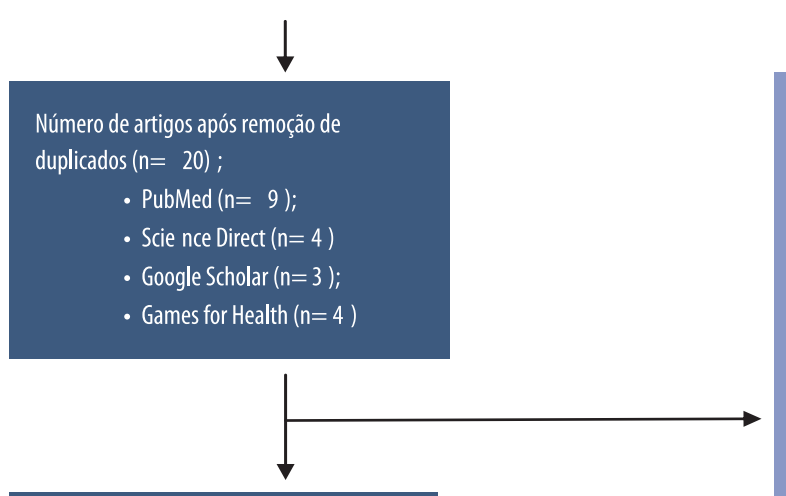

Número de artigos após seleção por resumos $(n=7)$

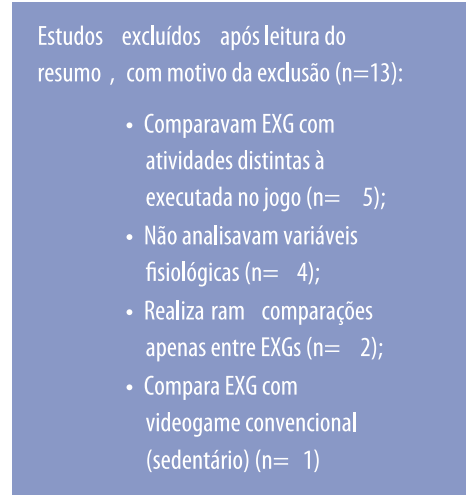

Número de artigos incluídos à revisão $(n=10)$ 
TABELA 1 - Descrição dos estudos incluídos

\begin{tabular}{|c|c|c|c|c|c|}
\hline Estudo & Delineamento & Participantes & Intervenção & Variáveis & Resultados \\
\hline $\begin{array}{l}\text { Warburton } \\
\text { et al. }{ }^{19}\end{array}$ & $\begin{array}{l}\text { Transversal, } \\
\text { medidas } \\
\text { repetidas, } \\
\text { contrabalanceado }\end{array}$ & $\begin{array}{l}\text { Adultos, } 7 \mathrm{H}, 7 \mathrm{M}, 24,6 \pm \\
4,2 \text { anos ((H) 25,9 } \pm 5,6 ; \\
\text { (M) } 23,4 \pm 2,1), 173,8 \pm \\
8,5 \mathrm{~cm}((\mathrm{H}) 179,9 \pm 5,0 ; \\
\text { (M) } 167,7 \pm 6,4), 70,1 \pm \\
15,0 \mathrm{~kg}((\mathrm{H}) 79,6 \pm 12,2 ; \\
\text { (M) } 60,6 \pm 11,3), \mathrm{e} \mathrm{IMC}= \\
23,1 \pm 4,7 \mathrm{~kg} / \mathrm{m}^{2} \text { ((H) } 25,6 \\
\pm 5,3 ; \text { (M) } 20,7 \pm 2,2) .\end{array}$ & $\begin{array}{l}15 \text { min pedalando em } \\
\text { bicicleta estacionária } \\
\text { (5 min a } 25 \% \text { da carga } \\
\text { máxima, } 5 \text { min de } \\
\text { intervalo, } 5 \text { min a } 50 \% \\
\text { da carga máxima, } \\
5 \text { min intervalo, } 5 \\
\text { min a } 75 \% \text { da carga } \\
\text { máxima) comparados } \\
\text { com o mesmo circuito } \\
\text { jogando GameBike } \\
\text { com os mesmos } \\
\text { percentuais de carga. }\end{array}$ & 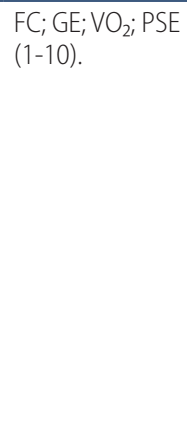 & $\begin{array}{l}\text { FC de reserva significantemente maior durante } \\
\text { o jogo ( } 26,0 \% \pm 18,0 \% \text { contra } 14,0 \% \pm 13,0 \%) \\
\text { nas cargas } 25,0 \% \text { e } 50,0 \% \text {, e não houve } \\
\text { diferença significativa para a carga } 75 \% \text {. } \mathrm{VO}_{2} \\
\text { significantemente maior durante o jogo }(34,0 \% \\
\pm 17,0 \% \text { contra } 18,0 \% \pm 12,0 \% \text { da capacidade } \\
\text { máxima) nas cargas } 25 \% \text { e } 50 \% \text {, porém não } \\
\text { houve diferença significativa para a carga } 75 \% \text {. GE } \\
\text { significantemente maior durante o jogo }(61,0 \% \\
\pm 41,0 \% \text { contra } 25,0 \% \pm 21,0 \%) \text { nas cargas } 25 \% \\
\text { e } 50 \% \text {, porém não houve diferença significativa } \\
\text { para a carga } 75 \%) \text {. PSE não teve diferença } \\
\text { significativa entre as atividades e cargas. }\end{array}$ \\
\hline Kraft et al. ${ }^{15}$ & $\begin{array}{l}\text { Transversal, } \\
\text { medidas } \\
\text { repetidas, } \\
\text { contrabalanceado }\end{array}$ & $\begin{array}{l}\text { Universitários, } 20 \mathrm{H}, 17 \mathrm{M} \\
(\mathrm{H}) 23,2 \pm 8,12 \text { anos, }(\mathrm{M}) \\
20,6 \pm 2,2 \text { anos, }(\mathrm{H}) 179,5 \\
\pm 8,0 \mathrm{~cm},(\mathrm{M}) 168,6 \pm 8,7 \\
\mathrm{~cm},(\mathrm{H}) 92,2 \pm 22,2 \mathrm{~kg} \\
\text { (M) } 68,8 \pm 13,6 \mathrm{~kg}\end{array}$ & $\begin{array}{l}30 \text { min pedalando } \\
\text { bicicleta estacionária } \\
\text { velocidade auto } \\
\text { selecionada assistindo } \\
\text { TV comparados com } \\
30 \text { minutos jogando }\end{array}$ & FC; PSE (1-10). & $\begin{array}{l}\text { FC média e pico foram significativamente } \\
\text { maiores para o jogo (média } 144,6 \pm 22,0 \text { contra } \\
126,6 \pm 20,0 \text { bpm; pico } 161,3 \pm 23,0 \text { contra } 144,6 \\
\pm 24,0 \text { bpm). Não houve diferença significativa } \\
\text { para PSE (jogo: } 4,6 \pm 1,7 ; \text { bicicleta: } 4,1 \pm 1,6 \text { ). }\end{array}$ \\
\hline
\end{tabular}

$\begin{array}{lll}\text { Douris et } & \text { Transversal, } & \text { Universitários, } 9 \mathrm{H} \mathrm{e} 12 \mathrm{M} \text {, } \\ \text { al. }{ }^{14} & \text { medidas } & 23,2 \pm 1,8 \text { anos, } 170,1 \pm \\ & \text { repetidas, } & 10,9 \mathrm{~cm}, 69,4 \pm 16,5 \mathrm{~kg} \text { e } \\ & \text { contrabalanceado } & \mathrm{IMC}=23,7 \pm 3,7 .\end{array}$

Haddock et Transversal, Escolares, $48 \mathrm{H}, 60 \mathrm{M}$, al. ${ }^{17}$ medidas $12,0 \pm 1,2$ anos, $154,0 \pm$ repetidas, $\quad 8,8 \mathrm{~cm}, 47,5 \pm 11,5 \mathrm{~kg} \mathrm{e}$ contrabalanceado $I M C=19,9 \pm 3,6 \mathrm{~kg} / \mathrm{m}^{2}$.

$\begin{array}{lll}\text { Griffin et } & \text { Transversal, } & \text { Adultos jovens, } 26 \mathrm{H}, 9 \\ \text { al. }^{16} & \text { medidas } & \mathrm{M}, 20,4 \pm 0,9 \mathrm{anos}, 175,8 \\ & \text { repetidas, } & \pm 5,3 \mathrm{~cm}, 71,0 \pm 7,0 \mathrm{~kg} \mathrm{e} \\ & \text { contrabalanceado } & \mathrm{IMC}=22,9 \pm 2,6 \mathrm{~kg} / \mathrm{m}^{2} .\end{array}$

Soltani et Transversal al. ${ }^{21}$

Monedero Transversal, et al. ${ }^{11}$ medidas repetidas, $1,7 \pm 0,1 \mathrm{~m} ; 72,6 \pm 10,9$

Naugle et Transversal, al. ${ }^{5}$ medidas repetidas
Adultos jovens, $60 \mathrm{H}$, $20 \pm 1$ anos; $1,79 \pm 0,0 \mathrm{~m}$; $73,9 \pm 14.6 \mathrm{~kg}$.
CatEye GB300, jogo

interativo com bicicleta estacionária.

30 min de caminhada em esteira $(3,5-5,64$ $\mathrm{km} / \mathrm{h}$ ) comparados com 30 min jogando FREERUN, no console Nintendo Wii.

Testes aeróbios de 1 milha PSE (1-10) de corrida/caminhada e PACER comparados com jogar Jackie Chan Action Run, jogo interativo em esteira.

30 min de sessão de fisioterapia convencional comparados com 30 minutos de fisioterapia com Wii Fit, do console Nintendo Wii.

Correr em esteira até a exaustão comparado com jogar Wii Fit Running até a exaustão (velocidades de corrida não foram mencionadas)

30 min pedalando bicicleta estacionária comparados com 30 min jogando Interactive Cycling Video Game, ambos com carga de $55 \%$ da potência máxima.

20 min pedalando em bicicleta estacionária com intensidade 11-13 PSE comparados com 20 minutos jogando Wii Cycling do Wii Fit no console Nintendo Wii.

FC; Duplo produto cardíaca $x$ (6-20). $\mathrm{VO}_{2} ; \mathrm{METS}$.

FC (frequência pressão arteria sistólica); PSE

PSE foi menor para o jogo $(3,8 \pm 1,9)$ do que para os testes de 1 milha de corrida/caminhada $(5,9 \pm 1,8)$ e PACER $(5,7 \pm 2,1)$. Houve correlação entre as três atividades $(r=-0,598$ a 0,312$)$.

PSE (1-10); GE; $\quad \mathrm{VO}_{2}$ foi significantemente maior para a sessão convencional ( 0,6 contra 0,1 L/min). GE significantemente maior para a sessão convencional (186,0 contra 146,5 J/kg/min). METs foram significantemente maiores para a sessão convencional (2,6 contra 2,1). Não houve diferença significativa para FC (jogo: $94 \pm 13$ bpm; real: $97 \pm 14$ bpm; $p=0,523$ ) Não houve diferença significativa na FC média entre as atividades (jogo: 124,9 $\pm 37,6$ bpm; real: $135,8 \pm 23,5$ bpm; $p=0,900$ )

FC; GE; $\mathrm{VO}_{2} ;$ PSE \% do VO2 reserva foi significantemente maior (6-20). durante o jogo $(68,2 \pm 9,2 \%$ contra $64,7 \pm 8,1 \%)$. GE foi maior durante o jogo $(505,8 \pm 75,2$ contra 487,4 $\pm 81,2 \mathrm{~J} / \mathrm{kg} / \mathrm{min})$. Não houve diferença significante no $\%$ de $F C$ de reserva $(72,5 \pm 10,4 \%$ contra $71,4 \pm$ $10,1 \%)$ e na PSE $(13,1 \pm 1,8$ contra $13,2 \pm 1,7)$.

FC; PSE (6-20). \%FC reserva foi significativamente maior para a bicicleta estacionária nos grupos Al e BI (jogo: 26,5-35,1\%; real: 49,1-63,4\%). PSE não teve diferença significativa entre as atividades e os grupos (jogo: 9,5-11,3; real: 11,6-12,6). $\mathrm{Bl}=11(4 \mathrm{H}, 7 \mathrm{M}, \mathrm{Al}=11$ $(3 \mathrm{H}, 8 \mathrm{M}),(\mathrm{Bl}) 20,7 \pm 1,2$ anos; (Al) 20,2 $\pm 0,9$ anos, (BI) $164,4 \pm 8,1 \mathrm{~cm}$, (Al) $165,2 \pm 10,0 \mathrm{~cm},(\mathrm{BI}) 68,0 \pm$ $10,7 \mathrm{~kg}$, (Al) $58,9 \pm 17,9 \mathrm{~kg}$. 


\begin{tabular}{|c|c|c|c|c|c|}
\hline Estudo & Delineamento & Participantes & Intervenção & Variáveis & Resultados \\
\hline $\begin{array}{l}\text { O'Donovan } \\
\text { et al. }{ }^{20}\end{array}$ & $\begin{array}{l}\text { Transversal, } \\
\text { medidas repetidas }\end{array}$ & $\begin{array}{l}\text { Universitários, } 5 \mathrm{H}, 13 \mathrm{M}, \\
21,9 \pm 2,0 \text { anos, } 172,9 \pm \\
7,9 \mathrm{~cm}, 69,4 \pm 7,4 \mathrm{~kg} \text { e } \\
\mathrm{IMC}=23,2 \pm 1,9 \mathrm{~kg} / \mathrm{m}^{2}\end{array}$ & $\begin{array}{l}\text { Teste progressivo em } \\
\text { esteira (Protocolo } \\
\text { Bruce) comparado com } \\
10 \text { min jogando Wii } \\
\text { Fit Free Jogging, do } \\
\text { console Nintendo Wii. }\end{array}$ & $\mathrm{FC}_{i} \mathrm{VO}_{2}$ & $\begin{array}{l}\text { FCmáx foi significantemente maior ( } p=0,002) \\
\text { durante o teste progressivo em esteira }(195,0 \\
\pm 6,0 \text { contra } 187,0 \pm 9,0 \mathrm{bpm}) . \mathrm{VO}_{2} \text { máximo foi } \\
\text { significantemente maior }(p=0,001) \text { durante } 0 \text { teste } \\
\text { progressivo em esteira }(46,8 \pm 10,1 \text { contra } 39,6 \pm 7,0 \\
\mathrm{mL} / \mathrm{min} / \mathrm{kg})\end{array}$ \\
\hline $\begin{array}{l}\text { Perusek et } \\
\text { al. }{ }^{18}\end{array}$ & $\begin{array}{l}\text { Transversal, } \\
\text { medidas } \\
\text { repetidas, } \\
\text { contrabalanceado }\end{array}$ & $\begin{array}{l}\text { Adultos jovens, } 15 \mathrm{H}, 14 \\
\mathrm{M}, 25,6 \text { anos, } 171,3 \mathrm{~cm} \text { e } \\
71,8 \mathrm{~kg} .\end{array}$ & $\begin{array}{l}10 \text { séries de } 3 \text { min } \\
\text { boxeando saco de } \\
\text { pancadas comparados } \\
\text { com } 10 \text { séries de } 3 \text { min } \\
\text { jogando Wii Boxing, } \\
\text { do jogo Wii Sports, do } \\
\text { console Nintendo Wii. }\end{array}$ & $\begin{array}{l}\text { FC; GE; PSE } \\
(6-20) .\end{array}$ & $\begin{array}{l}\text { FC foi significantemente maior }(p=0,001) \text { para socar } \\
\text { saco de pancadas }(156,0 \text { contra } 138,0 \text { bpm). PSE } \\
\text { foi significantemente maior }(p=0,001) \text { para socar } \\
\text { saco de pancadas }(13,8 \text { contra } 11,4) \text {. GE não foi } \\
\text { significativamente diferente }(p=0,078) \text { para ambos } \\
(241,0 \text { kcal para o saco de pancadas contra } 213,0 \mathrm{kcal} \\
\text { para o jogo). }\end{array}$ \\
\hline
\end{tabular}

FC: frequência cardíaca; FCmáx: frequência cardíaca máxima; bpm: batimentos por minuto; GE: Gasto energético; PSE: Percepção subjetiva de esforço; $\mathrm{VO}_{2}$ : Consumo de oxigênio; MET: Equivalente metabólico; $\mathrm{H}$ : homens; M: mulheres; Al: alta intensidade; Bl: baixa intensidade.

\section{Características das intervenções}

Todos estudos selecionados possuem delineamento experimental, nove com análise de medidas repetidas ${ }^{5,14-20}$, sendo sete deles contrabalanceados ${ }^{11,14-19} \mathrm{e}$ dois não foram contrabalanceados ${ }^{5,20}$. Apenas um estudo não utilizou medidas repetidas e não foi contrabalanceado ${ }^{21}$.

Ademais, nove estudos recrutaram sujeitos de ambos $\operatorname{sexos}^{5,14-20}$ e apenas um recrutou somente homens ${ }^{21}$. As amostras selecionadas foram compostas por universitários ${ }^{5,14,15,19}$, adultos jovens ${ }^{11,16,18,20,21}$ e escolares $^{17}$. Com exceção do estudo de Haddock et al. ${ }^{17}$, que foi realizado com escolares de 12,0 $\pm 1,2$ anos, os demais estudos utilizaram sujeitos adultos com idades entre $20 \pm 1,0 \operatorname{anos}^{21}$ e $26,6 \operatorname{anos}^{18}$.

Para as intervenções, seis estudos usaram o console Nintendo Wiiß $\circledR^{5,14,16,18,20,21} \mathrm{e}$ os outros quatro utilizaram sistemas não populares, como Interactive Cycling Video Game $^{11}$, CatEye GB300 ${ }^{15}$, GameBike ${ }^{19}$ e Jackie Chan Action Run ${ }^{18}$.

Nesse contexto, quatro estudos compararam EXG de ciclismo com bicicleta estacionária convencional ${ }^{5,11,15,19}$, quatro compararam EXG de corrida com correr em esteira ou pista $^{14,17,20,21}$. Ainda, um comparou EXG de boxe com treinar boxe em saco de pancadas ${ }^{18} \mathrm{e}$ um confrontou sessão de fisioterapia com EXG com sessão de fisioterapia convencional ${ }^{16}$.

Após o término, observamos que em frequência cardiaca (FC): três estudos encontraram valores superiores de FC durante prática com jogo em EXG. Kraft et al. ${ }^{15}$ encontraram valor médio de $144,6 \pm 22,0 \mathrm{bpm}$ para o jogo, contra $126,0 \pm 20,0$ bpm da atividade real, portanto $14,7 \%$ menor. Douris et al. ${ }^{14}$ encontraram valor máximo de 142,4 bpm para o EXG, contra 123,2 $\pm 13,7$ bpm da atividade convencional, logo $15,6 \%$ menor. Warburton et al. ${ }^{19}$ encontraram, para os percentuais de $25 \%$ e $50 \%$ da carga máxima dos cicloergômetros do EXG e convencional, valores médios de 128,7 $\pm 17,9 \mathrm{bpm}$ e 157,6 $\pm 20,4 \mathrm{bpm}$ para o jogo, contra 96,5 $\pm 12 \mathrm{bpm}$ e $135,9 \pm 15,9 \mathrm{bpm}$ na atividade real, ou seja, valores $33,4 \%$ e $16 \%$ menores, respectivamente. Além disso, três estudos não alcançaram diferenças significativas entre as atividades ${ }^{11,16,21}$, e três obtiveram valores maiores para a atividade real, com valores entre 49,1\% e 63,4\% da FC de reserva ${ }^{5}$, e valores máximos de 156 bpm $^{18}$ e 195,0 $\pm 6,0 \mathrm{bpm}^{20}$, maiores $13 \%$ e $15 \%$ respectivamente que o jogo.

$\mathrm{Na}$ análise estatística, não foi observada diferença significante na FC para as atividades convencionais ou em EXG, com média de diferença de -0,3 bpm (IC95\% $=-0,8-0,2 ; \mathrm{p}=0,248$ ) (Figura 2).

Seguidamente, em percepção subjetiva de esforço (PSE): quatro estudos não mostraram diferenças significativas para os valores de PSE entre o jogo e a atividade real ${ }^{5,11,15,19}$. Haddock et al. ${ }^{17}$ e Perusek et al. ${ }^{18}$ indicaram valores maiores para a ativi- 
dade real, ao passo que Douris et al. ${ }^{14}$ encontraram valores superiores com EXG na atividade Wii Fit "Free Run" quando comparada à atividade de corrida convencional $(12,7 \pm 6,3$ ua versus $10,1 \pm 3,3$ ua, $\mathrm{p}=0,014)$.

$\mathrm{Na}$ análise estatística, não foram percebidas diferenças significativas nos valores de PSE, com média de diferença de 0,3 (IC95\% = -0,2 - 0,8; p= 0,295) (Figura 3).

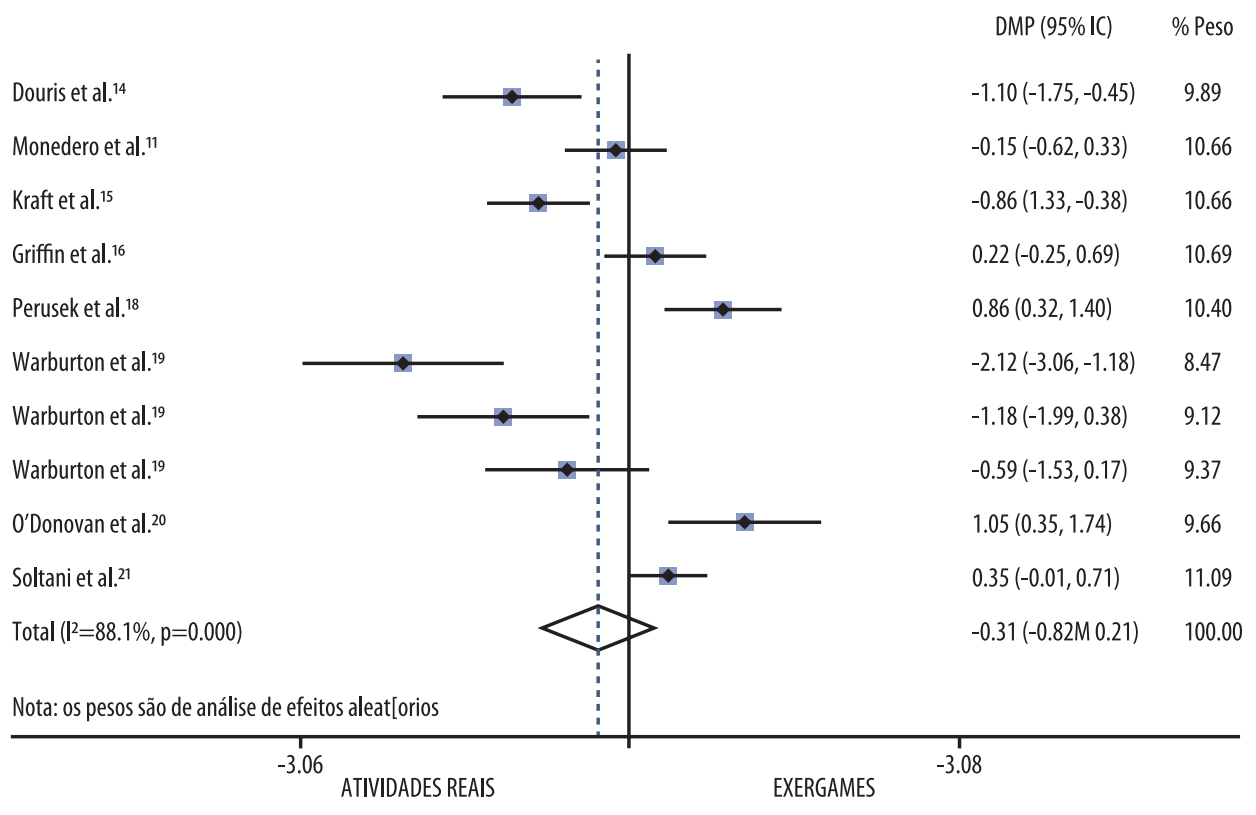

FIGURA 2 - Comparação dos dados de Frequência Cardíaca (FC) entre as atividades convencionais e exergames.

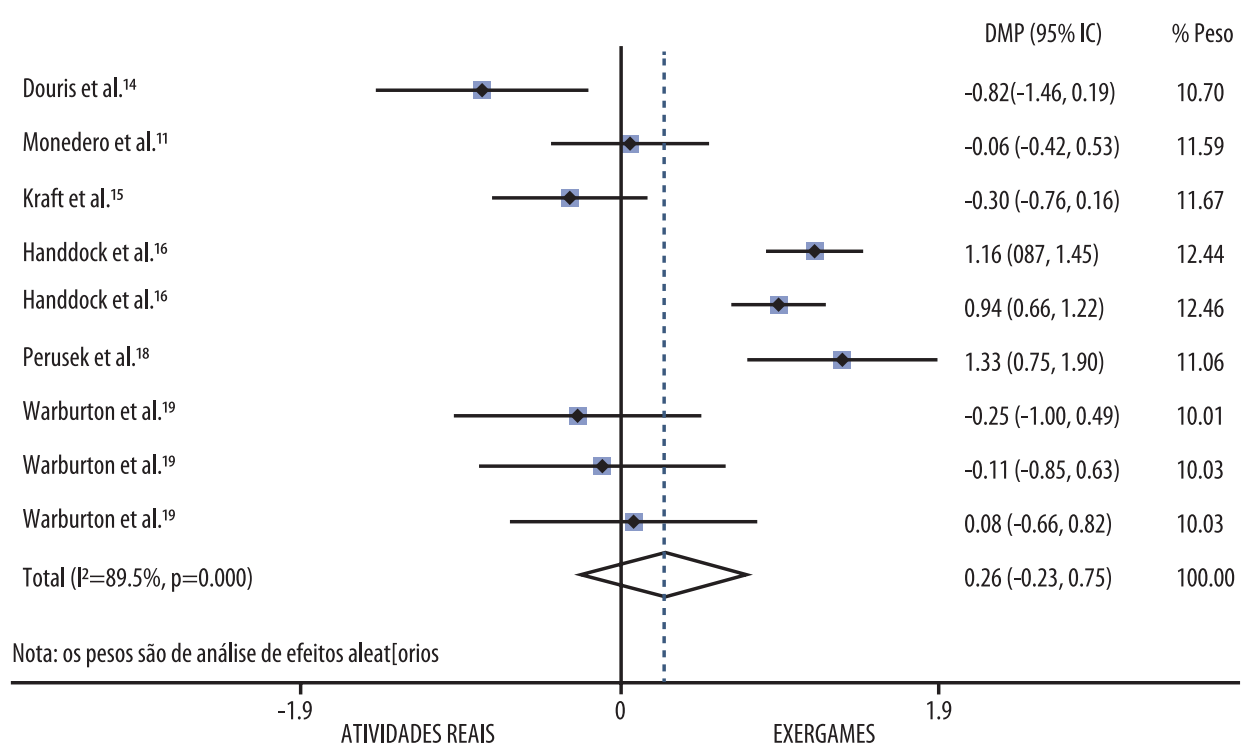

FIGURA 3 - Comparação dos dados de Percepção Subjetiva de Esforço (PSE) entre as atividades convencionais e exergames.

Já em gasto energético (GE): quatro estudos mensuraram GE e dois deles demonstraram valores maiores para o EXG. No primeiro, o GE foi de 505,8 $\pm 75,2$ $\left(\mathrm{J} \cdot \mathrm{kg}^{-1} \cdot \mathrm{min}^{-1}\right)$ para a atividade com EXG e 487,4 $\pm 81,2\left(\mathrm{~J} \cdot \mathrm{kg}^{-1} \cdot \mathrm{min}^{-1}\right)$ para a atividade convencional ${ }^{11}$, enquanto no segundo, $92,0 \pm 83,0 \mathrm{kcal}$ para a atividade com EXG e $63,5 \pm 66,9 \mathrm{kcal}$ para a atividade convencional ${ }^{19}$. O estudo um não encontrou diferenças significativas ${ }^{18}$, enquanto outro registrou valores estatisticamente maiores para a atividade real $\left(186,0 \text { versus } 146,5 \mathrm{~J} \cdot \mathrm{kg}^{-1} \cdot \mathrm{min}\right)^{16}$. 
$\mathrm{Na}$ metanálise não foram encontradas diferenças de GE entre as atividades convencionais e em EXG, com média de diferença de -0,2 (IC95\% = -0,9 - 0,6; p= $0,664) \mathrm{J}^{-1} \cdot \mathrm{kg}^{-1} \cdot \min$ (Figura 4).

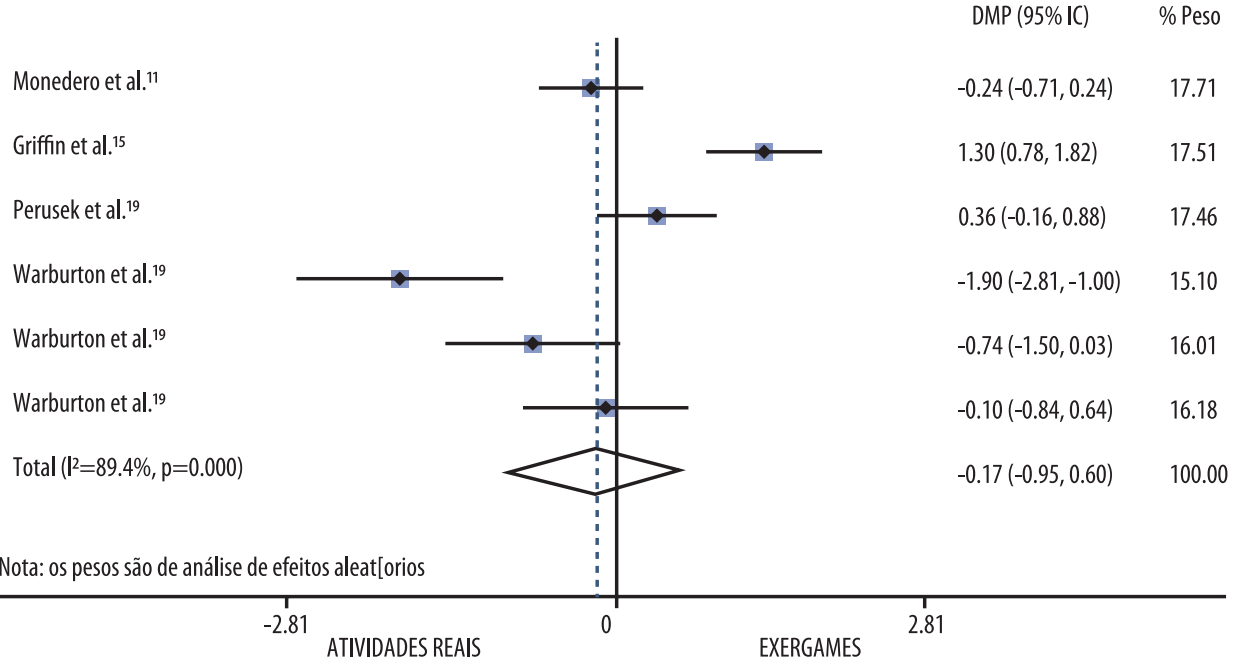

FIGURA 4 - Comparação dos dados de Gasto Energético (GE) entre as atividades convencionais e os exergames.

Em volume de oxigênio ( $V O)$ : dois estudos encontraram valores significativamente maiores no consumo de oxigênio. Adultos pedalando com média de consumo máximo de oxigênio de 44,0 $\pm 10,6\left(\mathrm{ml}^{-1} \cdot \mathrm{min}^{-1} \mathrm{~kg}\right)$ atingiram 34,0 $\pm 17,0 \%$ do VO2max na prática de EXG em cicloergômetro, contra $18,0 \pm 12,0 \%$ na prática com cicloergômetro convencional ${ }^{19}$. Com grupo populacional e atividade semelhante (cicloergômetro com e sem EXG), mas com $\mathrm{VO}_{2}$ max médio de $35,2 \pm 7,2 \mathrm{ml}^{-1} \cdot \mathrm{min}^{-1} \mathrm{~kg}$, chegou-se a $71,1 \pm 7,8 \%$ e $68,5 \pm 7,4 \%$ do máximo, respectivamente ${ }^{11}$. Outros dois estudos encontraram superioridade na atividade real, um comparando sessão de fisioterapia utilizando o console Nintendo Wii Fit com uma sessão convencional encontrou 0,5 \pm 0,2 $\mathrm{1}^{-1} \cdot \mathrm{min}$ versus $0,6 \pm 0,1 \mathrm{1}^{-1} \cdot \mathrm{min}^{16}$. Já outo confrontando o jogo Wii Fit Free Jogging com caminhada em esteira encontrou 44,9 $\pm 7,2 \mathrm{ml}^{-1} \cdot \mathrm{min}^{-1} \mathrm{~kg}$ versus $42,1 \pm 6,4 \mathrm{ml}^{-1} \cdot \mathrm{min}^{-1} \mathrm{~kg}^{20}$.

$\mathrm{Na}$ análise estatística não foram encontradas diferenças de $\mathrm{VO}$, com média de diferença de $-0,3(\operatorname{IC} 95 \%=-1,1-0,5 ; p=0,455) \mathrm{L} / \mathrm{min}$ (Figura 5).

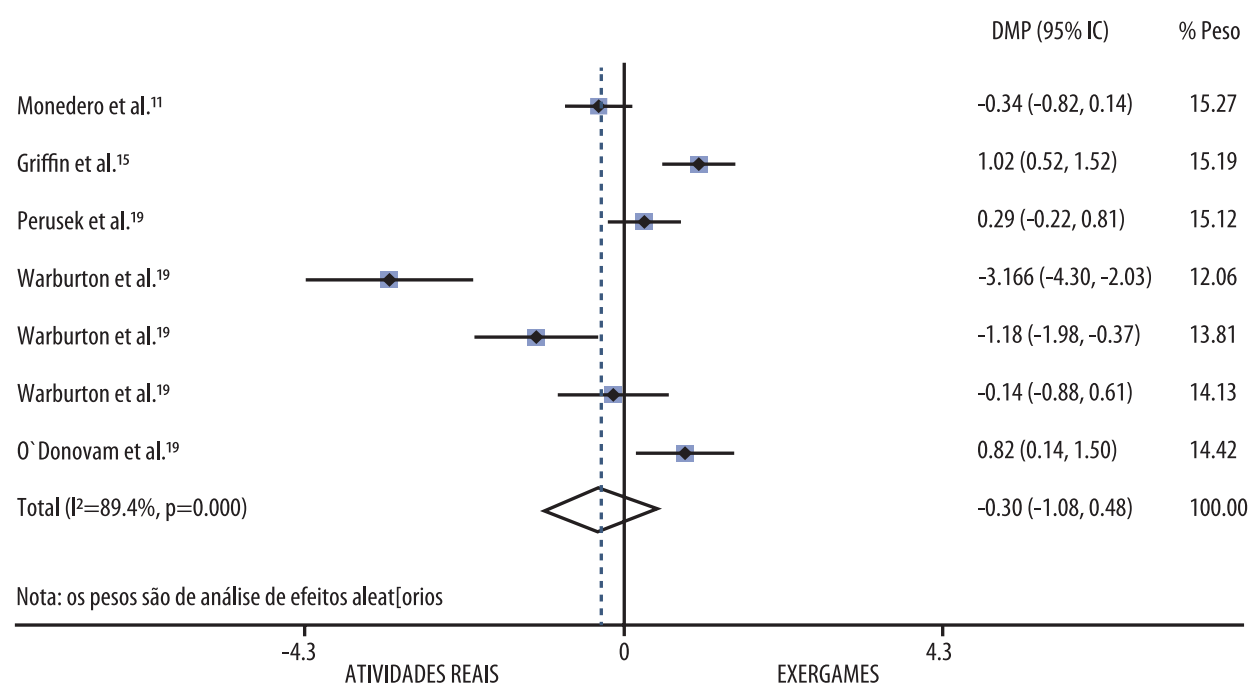

FIGURA 5 - Comparação dos dados de Volume de Oxigênio (VO2) entre as atividades convencionais e os exergames. 
Por fim, em equivalente metabólico (MET): apenas Griffin et al. ${ }^{16}$ analisaram METs e encontraram valores estatisticamente maiores durante atividade real (2,6 $\pm 0,4$ contra $2,1 \pm 0,5$ METs).

Ao serem submetidos à escala de qualidade metodológica PEDro, quatro estudos obtiveram pontuação $7 / 11^{5,14,17,18}$ e seis estudos obtiveram pontuação seis ${ }^{11,15,16,19,20,21}$. Vale destacar ainda, que a natureza da intervenção dos estudos desta revisão não permitia que os itens cinco, seis e sete da escala PEDro fossem atendidos. Sendo assim, a pontuação detalhada e discriminada por item da escala PEDro se encontra na tabela 2.

TABELA 2 - Qualidade metodológica dos estudos segundo a Escala PEDro.

\begin{tabular}{|c|c|c|c|c|c|c|c|c|c|c|c|c|}
\hline \multirow{2}{*}{ Estudo } & \multicolumn{12}{|c|}{ Itens da Escala PEDro } \\
\hline & $\# 1$ & $\# 2$ & \#3 & $\# 4$ & \#5 & $\# 6$ & \#7 & \#8 & $\# 9$ & $\# 10$ & $\# 11$ & Total \\
\hline Warburton et al. ${ }^{19}$ & 0 & 1 & 0 & 1 & 0 & 0 & 0 & 1 & 1 & 1 & 1 & $6 / 11$ \\
\hline Kraft et al. ${ }^{15}$ & 1 & 0 & 0 & 1 & 0 & 0 & 0 & 1 & 1 & 1 & 1 & $6 / 11$ \\
\hline Douris et al. ${ }^{14}$ & 1 & 1 & 0 & 1 & 0 & 0 & 0 & 1 & 1 & 1 & 1 & $7 / 11$ \\
\hline Haddock et al. ${ }^{17}$ & 1 & 1 & 0 & 1 & 0 & 0 & 0 & 1 & 1 & 1 & 1 & $7 / 11$ \\
\hline Griffin et al. ${ }^{16}$ & 1 & 0 & 0 & 1 & 0 & 0 & 0 & 1 & 1 & 1 & 1 & $6 / 11$ \\
\hline Soltani et al. ${ }^{21}$ & 1 & 0 & 0 & 1 & 0 & 0 & 0 & 1 & 1 & 1 & 1 & $6 / 11$ \\
\hline Monedero et al..11 & 1 & 0 & 0 & 1 & 0 & 0 & 0 & 1 & 1 & 1 & 1 & $6 / 11$ \\
\hline Naugle et al..$^{5}$ & 1 & 1 & 0 & 1 & 0 & 0 & 0 & 1 & 1 & 1 & 1 & $7 / 11$ \\
\hline O'donovan et al. ${ }^{20}$ & 1 & 0 & 0 & 1 & 0 & 0 & 0 & 1 & 1 & 1 & 1 & $6 / 11$ \\
\hline Perusek et al. ${ }^{18}$ & 1 & 1 & 0 & 1 & 0 & 0 & 0 & 1 & 1 & 1 & 1 & $7 / 11$ \\
\hline
\end{tabular}

Um ponto foi dado para cada resposta "sim", nenhum ponto (zero) foi dado para respostas "não".

*NOTA: Os pontos foram concedidos SOMENTE quando o critério foi claramente satisfeito.

Houve indicativo de alta heterogeneidade estatística para os desfechos (FC: $\mathrm{I}^{2}=$ 88,1\%, p <0,001; PSE: $I^{2}=89,5 \%, p<0,001$; GE: $I^{2}=89,4 \%, p<0,001$ e VO $: I^{2}=90,4 \%$, $\mathrm{p}<0,001$.

\section{Discussão}

O presente estudo teve por objetivo realizar revisão sistemática da literatura com metanálise dos estudos que compararam efeitos fisiológicos agudos da prática de EXG com a prática de atividades reais da mesma temática dos jogos. O principal achado da presente investigação foi que não houve diferença entre os resultados dos estudos considerando os efeitos fisiológicos da prática de EXG das mesmas atividades convencionais. Apesar dos EXG não serem novidades, são escassos os estudos que comparem demandas fisiológicas da sua prática com as mesmas atividades reais, quando praticadas de modo convencional ${ }^{18}$. Pesquisas prévias compararam atividades em EXG, com tarefas distintas, sem nenhuma relação com o que é executado no console ${ }^{22,23}$. Portanto, o presente estudo tem, como ponto forte, a análise de atividades semelhantes realizadas em contextos distintos - com e sem presença de EXG.

Sabe-se que as demandas variam de acordo com o jogo e com a atividade real praticada $^{1}$. Reconhece-se que a natureza das atividades executadas é distinta, o que justifica os achados distintos entre estudos. No entanto, como as comparações foram pareadas considerando as mesmas atividades realizadas de dois modos, esta aparente limitação não se consiste como problema metodológico. 
Entres os estudos que comparavam atividades semelhantes também não houveram diferenças para diversas variáveis, o que, possivelmente, pode ser explicado pelas características metodológicas distintas entre eles. Nos estudos com bicicleta Monedero et al. ${ }^{11}$ realizaram teste de consumo máximo de oxigênio com todos sujeitos e os fizeram pedalar a $55 \%$ da potência máxima durante a atividade real e durante o jogo. Kraft et al. ${ }^{15}$, além de utilizarem intensidade autosselecionada, solicitaram que os indivíduos realizassem os testes em duplas e assistindo televisão durante a atividade real. Naugle et al. ${ }^{5}$ solicitaram que durante a atividade real os indivíduos pedalassem em uma cadência que correspondesse a 11-13 pontos na escala de Borg (atividade moderada), enquanto no jogo com EXG não havia relato de recomendação sobre a intensidade. Enquanto que Warburton et al. ${ }^{19}$ concretizaram teste incremental de carga nos sujeitos e, durante o jogo e a atividade real, empregaram protocolo incremental de três níveis da carga máxima - 25\%, 50\% e 75\% da potência máxima (W) mensurada previamente em teste incremental. Estas diferenças podem justificar os achados não semelhantes entre as variáveis estudadas.

Nos jogos que envolveram corrida Douris et al. ${ }^{14}$ compararam 30 minutos de jogo (FREE RUN, console Nintendo Wii) com 30 minutos de caminhada com velocidade entre $3,5 \mathrm{~km} / \mathrm{h}$ e $5,64 \mathrm{~km} / \mathrm{h}$, enquanto Haddock et al. ${ }^{17}$ conferiram o jogo em esteira Jackie Chan Action Run com dois testes de condicionamento aeróbio (1 milha de caminhada/corrida e PACER). Já O'Donovan et al. ${ }^{20}$ confrontaram $10 \mathrm{mi}$ nutos de jogo (Wii Fit Free Jogging, do console Nintendo Wii) com teste progressivo em esteira (protocolo Bruce), enquanto Soltani et al. ${ }^{21}$ comparou correr em esteira até a exaustão, em velocidade não mencionada, com jogar Nintendo Wii Fit Running também até a exaustão. Houveram desfechos distintos para FC e PSE, e os achados de $\mathrm{VO}_{2}$ não são comparáveis, pois O’Donovan et al. ${ }^{20}$ foram os únicos a analisá-los.

Apesar de não haver diferenças nos achados, seja pelo fato das atividades serem distintas ou das particularidades metodológicas, outros podem ser relevantes. Nos estudos com corrida a atividade executada no jogo teve baixa correlação ( $\mathrm{r}=$ -0,59 a 0,312), o que indica que o EXG pode não ser um bom substituto à atividade real ${ }^{17}$. No entanto, nos jogos com bicicleta, apesar de achados distintos para FC, não houveram diferenças significativas para PSE, o que indica que os EXG podem interferir na percepção de esforço em decorrência da imersão na prática ${ }^{15}$. Tal achado também foi evidenciado no estudo com boxe, que apresentou valores menores de PSE para o jogo, mesmo com valores maiores de GE, 213,0 \pm 68,4 kcal versus $240,6 \pm 83,5 \mathrm{kcal}^{18}$.

Uma das limitações deste estudo foi a variedade inerente de atividades e de metodologias utilizadas nos manuscritos selecionados, que podem prejudicar a comparação entre os desfechos. Mesmo assim, reconhece-se que existem muitas opções de prática associadas aos EXG, o que aumentam as possibilidades de intervenções. Para superar isto, buscamos comparar apenas atividades semelhantes do ponto de vista motor e estrutural. Também, a variabilidade entre os estudos pode ter ocasionado a alta heterogeneidade estatística encontrada. Para lidar com isto, optou-se por conduzir as análises por modelo de efeitos aleatórios, que considera a variabilidade causada pela diferença metodológica de estudos distintos ${ }^{24,25}$. Outra opção seria realizar novas análises excluindo estudos que poderiam estar interferindo no resultado ${ }^{24,25}$. Porém, a partir de inspeção visual dos resultados, não fica claro quais estudos deveriam ser excluídos da análise, já que os dados estão relativamente bem distribuídos entre atividades reais e EXG. Outra limitação se associa ao fato de alguns estudos utilizarem consoles não populares ${ }^{11,15,18,19}$, o que prejudica a validade externa dos achados. Sabendo que os EXG são capazes 
de atingir as recomendações do American College of Sports Medicine (ACSM) de atividade física moderada ${ }^{8}$, como fora observado em alguns dos estudos ${ }^{9,10,18}$, que possuem bom nível de aderência entre pessoas sedentárias ${ }^{19}$ e são eficazes para reabilitação ${ }^{16}$. Dessa maneira, sugere-se que estudos futuros comparem as diferenças fisiológicas entre a prática virtual e a real, principalmente utilizando atividades e consoles de fácil acesso à população em geral.

Como conclusão, pontua-se que não há diferenças significativas entre as demandas das variáveis FC, PSE, VO e GE, em atividades com EXG e as mesmas atividades realizadas de maneira convencional.

\section{Declaração de conflito de interesses}

Os autores declaram não haver nenhum conflito de interesses inerente à realização deste estudo.

\section{Contribuiç̧ão dos autores}

B. B. Vasconcelos (0000-0003-0019-4493) participou da concepção do estudo, coleta de dados e redação do estudo. A. Formalioni (0000-0002-2277-9997) participou da concepção do estudo, coleta de dados e redação do estudo. L. M. Galliano (0000-0002-5599-0377) participou da análise estatística e da redação do estudo. C. A. O. Vaghetti (0000-0003-2584-7761) participou da redação do estudo. F. B. Del Vecchio (0000-0003-3771-9660) participou da concepção e da redação do estudo. Todos os autores aprovaram a versão final a ser publicada.

\section{Referências}

1. Bailey BW, Mcinnis K. Energy Cost of Exergaming: A comparison of the energy cost of 6 forms of exergaming. Arch Pediatr Adolesc Med. 2011;165:597-602.

2. Daley AJ. Can Exergaming Contribute to Improving Physical Activity Levels and Health Outcomes in Children? Pediatrics. 2009;124:763-71.

3. Finco MD, Reategui E, Zaro MA, Sheeran DD, Katz l. Exergaming as an Alternative for Students Unmotivated to Participate in Regular Physical Education Classes. IJGBL. 2015;5:1-10.

4. Bateni H. Changes in balance in older adults based on use of physical therapy vs. the Wii Fit gaming system: a preliminary study. Physiotherapy. 2012;98:211-16.

5. Naugle KE, Naugle KM, Wikstrom EA. Cardiovascular and affective outcomes of active gaming: Using the Nintendo Wii as a cardiovascular training tool. J Strength Cond Res. 2014;28:443-51.

6. Bronner S, Pinsker R, Naik R, Noah JA. Physiological and psychophysiological responses to an exer-game training protocol. J Sci Med Sport. 2016;19:267-71.

7. Gao Z, Zhang T, Stodden D. Children's physical activity levels and psychological correlates in interactive dance versus aerobic dance. J Sport Health Sci. 2013;2:146-51.

8. Garber CE, Blissmer B, Deschenes MR, Franklin B a, Lamonte MJ, Lee I-M, et al. American College of Sports Medicine position stand. Quantity and quality of exercise for developing and maintaining cardiorespiratory, musculoskeletal, and neuromotor fitness in apparently healthy adults: guidance for prescribing exercise. Med Sci Sports Exerc. 2011;43:1334-59.

9. Miyachi M, Yamamoto K, Ohkawara K, Tanaka S. METs in adults while playing active video games: a metabolic chamber study. Med Sci Sports Exerc. 2010;42:1149-53.

10. Graves LEF, Ridgers ND, Williams K, Stratton G, Atkinson G, Cable NT. The physiological cost and enjoyment of Wii Fit in adolescents, young adults, and older adults. J Phys Act Health. 2010;7:393-401.

11. Monedero J, Lyons EJ, O'gorman DJ. Interactive video game cycling leads to higher everny expenditure and is more enjoyable than conventional exercise in adults. PLoS One. 2014;10:e0118470.

12. Moher D, Liberati A, Tetzlaff J, Altman DG. Preferred reporting items for systematic 
reviews and meta-analyses: the PRISMA statement. Ann Intern Med. 2009;151:264-9.

13. Maher CG, Sherriington C, Herbert RD, Moseley AM, Elkins M. Reliability of the PEDro Scale for Rating Quality of Randomized Controlled Trials. Phys Ther. 2003;83:713-21.

14. Douris PC, Mcdonalds B, Vespi F, Kelley NC, Herman 1. Comparison between Nintendo Wii Fit Aerobics and Traditional aerobic exercise in sedentary young adults. J Strength Cond Res. 2012;1052-7.

15. Kraft JA, Russel WD, Bowman TA, Selsor ICW, Foster GD. Heart rate and perceived exertion during self-selected intensities for exergaming compared to traditional exercise in college-age participants. J Strength Cond Res. 2011;25:1736-42.

16. Griffin M, Shawis T, Impson R, Shanks J, Taylor MJ. Comparing the energy expenditure of Wii Fit-Based Therapy versus Traditional Physiotherapy. Games Health J. 2013;2:229-34.

17. Haddock B, Siegel S, Costa P, Jarvis S, Klug N, Medina E, et al. Fitness Assessment Comparison Between the "Jackie Chan Action Run" Videogame, 1-Mile Run/Walk, and the PACER. Games Health J. 2012;1:223-7.

18. Perusek K, Sparks K, Little K, Motley M, Pattersom S, Wieand J. A comparison of energy expenditure during "Wii Boxing" versus Heavy Bag Boxing in young adults. Games Health J. 2014;3:21-4.

19. Warburton DE, Sarkany D, Johnson M, Rhodes RE, Whitford W, Esch BT, et al. Metabolic requirements of interactive video game cycling. Med Sci Sports Exerc. 2009; 41:920-6.

20. O'donovan C, Gormley J, Hussey JM. The potential of "Wii Fit Free Jogging” as an exercise test. Games Health J. 2014;3:296-302.

21. Soltani P, Salesi M. Effects of Exergame and music on acute exercise responses to graded treadmill running. Games Health J. 2013;2:75-80.

22. Perron RM, Grahan CA, Hall EE. Comparison of Physiological and Psychological Responses to Exergaming and Treadmill Walking in Healthy Adults. Games Health J. 2012;1:411-5.

23. Bonetti AJ, Drury DG, Danoff JV, Miller TA. Comparison of acute exercise responses between conventional video gaming and isometric resistance exergaming. J Strength Cond Res. 2010;24:1799-803.

24. Rodrigues C, Ziegelmann P. Metanálise: um guia prático. Revista HCPA. 2010;30:435-46.

25. Sousa MR, Ribeiro ALP. Revisão sistemática e meta-análise de estudos de diagnóstico e prognóstico: um tutorial. Arq Bras Cardiol. 2009;29:241-51.

Endereço para

Correspondência

Breno Berny Vasconcelos

brenobvasc@gmail.com
Rua Doutor Francisco Simões, 243

Bairro Fragata, Pelotas, RS

CEP 96030-630
Recebido

Revisado

$28 / 11 / 2016$

$02 / 03 / 2017$

$18 / 04 / 2017$

$26 / 04 / 2017$

Aprovado $\quad 22 / 05 / 2017$ 